\title{
Proposed Technologies for Solving Future 5G Heterogeneous Networks Challenges
}

\author{
Isra Sitan Al-Qasrawi \\ Al-balqa' Applied University, \\ AL-Huson University College \\ Department of Information Technology \\ Irbid, Jordan
}

\begin{abstract}
The evolution towards $5 \mathrm{G}$ mobile communication networks will be characterized by increasing number of wireless devices and service complexity, while the requirement to access mobile services will be essential.

This paper presents an overview of the evolution of wireless networks, and focus on future mobile communication generation (5G) with its requirements, Challenges and Services. In addition, the paper proposes a heterogeneous architecture for $5 \mathrm{G}$ networks. The key ideas for each of the technologies are stated, along with the potential impact on $5 \mathrm{G}$ networks architecture.
\end{abstract}

The proposed architecture key elements such as Small cells, Massive MIMO, mm-waves, D2D communication, fullduplex communication, energy harvesting, Cloud-RAN and Wireless Network Virtualization, all of these technologies serve together to ensure users with Quality of service (QoS) requirement in a spectrum \& energy efficient manner.

\section{General Terms}

Heterogeneous Networks, Technology, Wireless, Challenges.

\section{Keywords}

5G networks, wireless cellular networks, 5G networks, 5G heterogeneous network architecture, small cells, D2D communications, Massive MIMO, mm-wave, C-RAN, energy harvesting.

\section{INTRODUCTION}

Revolution and evolution of Mobile wireless industry has started since early 1970 While mobile communication industry has witnessed huge growth since mid 1990's. The wide spread of cellular communications have led to develop new wireless systems and standards, besides mobile voice calls. The four key benefits of wireless technology are as under:

- Increased efficiency: High technology communication systems lead to faster transfer of information within business and between customers.

- Rarely out of touch: No need to carry cables or adapters in order to access office Networks.

- Greater flexibility for users: Wireless workers in the office can be networked without sitting at dedicated PC's.

- Reduced Cost: Wireless networks are mostly cheaper to install and maintain than wired networks [1].

One of the wireless technologies is wireless ad hoc network. Wireless Ad-hoc networks are considered as decentralized wireless networks, where they do not rely on a pre-established infrastructure. Nodes in wireless ad hoc networks are small, with low capacity in energy and storage [2]. These limitations exist too in cellular devices where the proposed technologies for $5 \mathrm{~g}$ heterogeneous network must be applied. These limitations and challenges must be taken in consideration to determine the proper technologies in 5G networks.

\subsection{The Evolution of Wireless Cellular Systems}

Nearly, a new mobile generation emerges every ten years or so. Until now, four generations of cellular communication systems have been adopted.

The first analog mobile networks appeared in 1980, second generation digital technology GSM followed in the 1991, 3G arrived at the turn of the century in 2001, and 4G LTE-A began rolling out in 2008 [3]. The evolution from $1 \mathrm{G}$ to $5 \mathrm{G}$ is summarized in Table 1 and Figure 1.

Each generation has set out to fix the drawbacks of its previous generation, $2 \mathrm{G}$ fixed the security weaknesses of analogue telephony in $1 \mathrm{G}$, while $3 \mathrm{G}$ was emerged to sort out GSM's lack of mobile data in $2 \mathrm{G}$, and $4 \mathrm{G}$ was needed to finally make consuming data less than before. Now, the industry of wireless technology hopes that $5 \mathrm{G}$ will solve problems we don't have today, but those that could hold us back years in the future.

First generation (1G) refers to the first generation of wireless telephone technology which was first introduced in 1980 and completed in early $1990 \mathrm{~s}$ with peed up to $2.4 \mathrm{kbps}$. Its network use Analog Signals and allows voice calls only.

Second generation (2G) refers to the second generation which is based on Global System Mobile (GSM). 2G network uses digital signals with digital multiple access technology, such as Code Division Multiple Access (CDMA) and Time Division Multiple Access (TDMA). This generation compared to $1 \mathrm{G}$ provides better quality and capacity, with more data rate speed up to $64 \mathrm{kbps}$. It enables services such as text messages, picture message and Multimedia Messaging Service (MMS).

2.5G refers to a technology between the second generation $(2 \mathrm{G})$ and the third generation (3G). It describes $2 \mathrm{G}$ systems that have implemented packet switched domain with circuit switched domain, with data transfer rate speed up to $144 \mathrm{kbps}$ and provides more services such as send and receive e-mail messages and web browsing.

Third generation (3G) refers to third generation of wireless telephone technology which was introduced in year 2001. It has large capacity and broadband capabilities, so data transfer rate speed increased from $144 \mathrm{kbps}-2$ mbps. This 
accommodates web-based applications and audio and video files such as video conferencing and Mobile TV. In this generation, phones were converted to smart phones.

3.5G refers to a technology between the third generation (3G) and the fourth generation (4G). $3 \mathrm{G}$ involves the introduction and utilization of different technologies such as: Wideband Code Division Multiple Access (WCDMA), Code Division Multiple Access (CDMA) and Universal Mobile Telecommunications Systems (UMTS). The evolving technologies like High Speed Uplink/Downlink Packet Access (HSUPA/HSDPA) and Evolution-Data Optimized (EVDO) has made an intermediate wireless generation between $3 \mathrm{G}$ and $4 \mathrm{G}$ named as $3.5 \mathrm{G}$ with improved data transfer rate of 5-30 Mbps [4].

Fourth generation (4G) refers to fourth generation which is generally referred as the descendant of the $3 \mathrm{G}$ and $2 \mathrm{G}$ standards. 3rd Generation Partnership Project (3GPP) is standardizing Long Term Evolution (LTE) Advanced as 4G standard along with Mobile Worldwide Interoperability for Microwave Access (WIMAX). This generation is evolving to provide IP connectivity for diverse applications and services.

4G supports packet switched network that allows mobile's access to dynamic information with data rate up to $1 \mathrm{Gbps}$. It is designed to improve cost efficiency, optimize network performance, and facilitate the use of market IP-based services. Voice, data and multimedia will be provided to users on every time and everywhere. There is wide range of Applications that use 4G network such as High Definition TV content, mobile TV, Digital Video Broadcasting (DVB), and video chat.

Fifth generation (5G) refers to fifth generation of wireless networks, which can be a complete wireless communication based on IPv6 protocol to provide unlimited access to information and the ability to share data anywhere, anytime by anyone for the benefit of the world, and is called also Real world wireless or World Wide Wireless Web (WWWW). The main aims of $5 \mathrm{G}$ are the Implementation of massive capacity and massive connectivity and providing several set of services, applications including camera, MP3 recording, video player, large phone memory, dialing speed, audio player and much more one can never imagine. Multiple applications will be accessed at one time with full security and finest Quality of
Service (QoS). The amazing features of 5G [5] will make it the main wireless network which will be used all over the world. The most important features of $5 \mathrm{G}$ networks are:

- less traffic, low-cost, bidirectional bandwidth

- availability of network across the world

- $\quad$ its terminals have software-defined radios

- $\quad$ connectivity speed up to $25 \mathrm{Mbps}$

- data bandwidth more than $1 \mathrm{~GB}$

- $\quad$ large broadcasting of data in Gigabit

- $\quad$ supports virtual private network

- $\quad$ provides hundreds of channels without streaming

- Remote diagnostics

- Very high uploading and downloading speed

- uses different modulation techniques and errorcontrol techniques

The main differences of $5 \mathrm{G}$ compared to $4 \mathrm{G}$ are greater spectrum allocations, longer battery life, higher bit rates in larger portions of the coverage area, higher total capacity for many users at same time in both licensed and unlicensed spectrum, lower outage probability, and lower infrastructure costs. The main networks of $5 \mathrm{G}$ will be based on mm-wave wireless connections instead of copper and fiber which will allow rapid deployment and mesh-like connectivity with cooperation between base stations.

5G networks will not be based on previous technologies like switching nor routing anymore. They will be more flexible, and work on Heterogeneous Networks, and able to be evolved more easily than the traditional networks.

$5 \mathrm{G}$ mobile networks must address six challenges that are not effectively addressed by 4G: higher data rate, higher capacity, massive device connectivity, lower End to End latency, reduced cost and consistent Quality of Experience provisioning [6], [7]. Figure 2 shows challenges and potential facilitators to address them [8].

Table 1. The Evolution of Wireless networks

\begin{tabular}{|c|c|c|c|c|c|c|}
\hline Generation & Started at & technology & Data rates & $\begin{array}{c}\text { Main } \\
\text { network }\end{array}$ & Handover & $\begin{array}{c}\text { Sub } \\
\text { generation }\end{array}$ \\
\hline $1 \mathrm{G}$ & 1980 & $\begin{array}{c}\text { Analog } \\
\text { wireless }\end{array}$ & $2 \mathrm{kbps}$ & PSTN & Horizontal & $1 \mathrm{G}$ only \\
\hline $2 \mathrm{G}$ & 1991 & $\begin{array}{c}\text { Digital } \\
\text { wireless, } \\
\text { GPRS, EDEG }\end{array}$ & $\begin{array}{l}10 \mathrm{kbps} \text { to } \\
500 \mathrm{kbps}\end{array}$ & $\begin{array}{c}\text { PSTN, } \\
\text { GSM, } \\
\text { WCD }\end{array}$ & Horizontal & $\begin{array}{l}2.5 \mathrm{G} \\
2.75 \mathrm{G}\end{array}$ \\
\hline $3 \mathrm{G}$ & 2001 & $\begin{array}{l}\text { Broad Band } \\
\text { IP Tech }\end{array}$ & $\begin{array}{l}400 \mathrm{kbps} \text { to } \\
30 \mathrm{mbps}\end{array}$ & $\begin{array}{c}\text { Packet, } \\
\text { GSM, } \\
\text { TDMA }\end{array}$ & $\begin{array}{l}\text { Horizontal } \\
\& \text { Vertical }\end{array}$ & $\begin{array}{l}3.5 \mathrm{G} \\
3.75 \mathrm{G}\end{array}$ \\
\hline $4 \mathrm{G}$ & 2008 & LTE, Wi-max & $\begin{array}{c}200 \mathrm{mbps} \text { to } \\
1 \mathrm{gbps}\end{array}$ & Internet & $\begin{array}{l}\text { Horizontal } \\
\text { \& Vertical }\end{array}$ & 4G only \\
\hline $5 \mathrm{G}$ & Will start 2020 & LTE, Wi-max & $\begin{array}{l}\text { Higher than } \\
\text { 1Gbps }\end{array}$ & Internet & $\begin{array}{l}\text { Horizontal } \\
\text { \& Vertical }\end{array}$ & $\begin{array}{c}5 \mathrm{G} \text { till } \\
\text { now }\end{array}$ \\
\hline
\end{tabular}




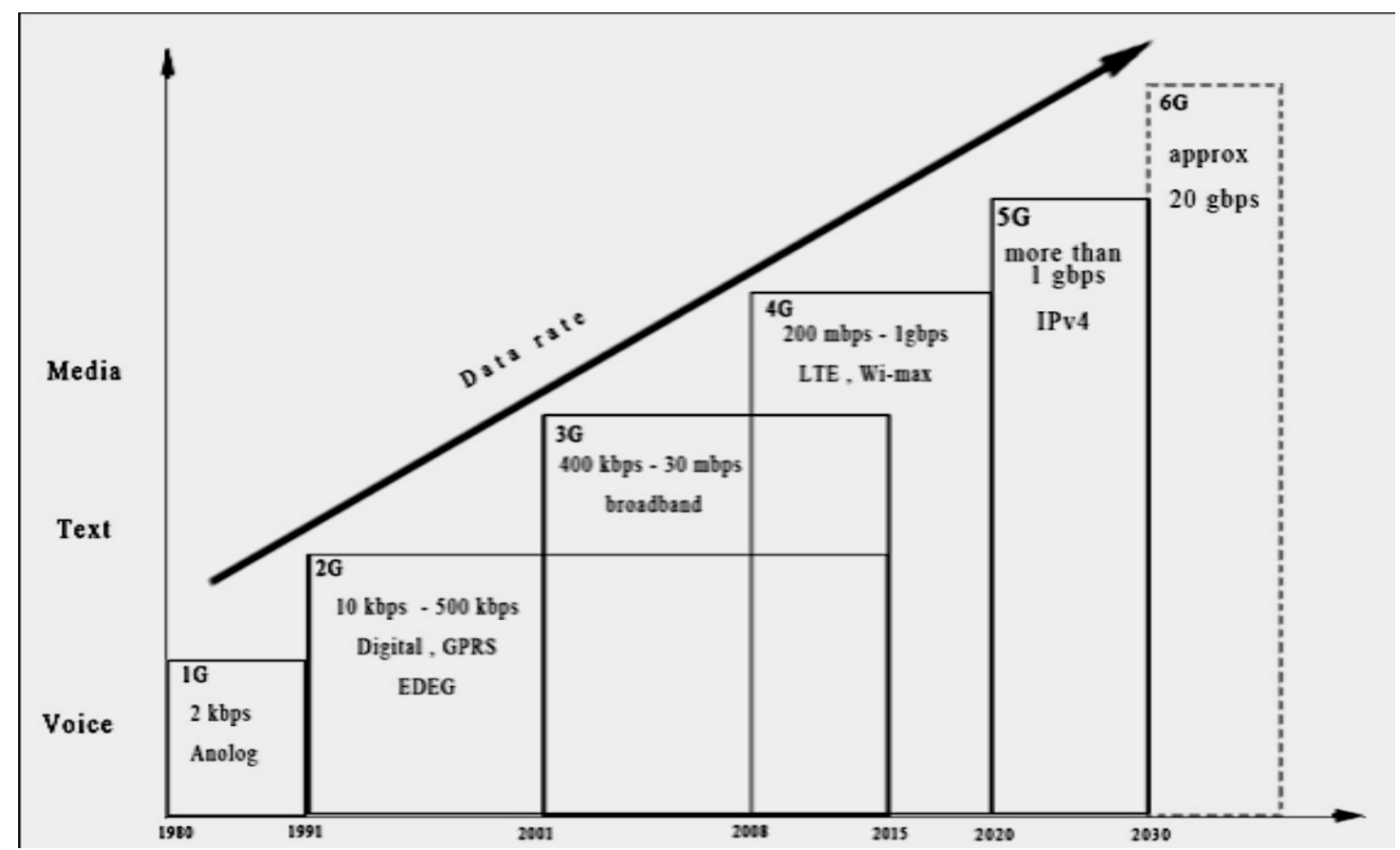

Figure 1 Evolution of Wireless Networks

\section{5G HETEROGENEOUS MULTI-TIER NETWORKS ARCHITECTURE}

To overcome the challenges of $5 \mathrm{G}$ system, a radical change in the strategy of designing the $5 \mathrm{G}$ network architecture is needed. The new architecture must address the fundamental requirements of $5 \mathrm{G}$ networks which are they:

a) Capabilities for supporting massive capacity and massive connectivity.

b) Providing Support for an increasingly various set of services, application and users.

c) Flexible and efficient use of all available noncontiguous spectrums in different networks.

The main technologies will be present in the architecture to address these requirements can be classified as follows [9-11]:

- Intensification of existing mobile networks with the massive addition of small cells, and presenting peerto-peer (P2P) communication such as: device-todevice [D2D] and machine-to-machine $[\mathrm{M} 2 \mathrm{M}]$ in multi-tier heterogeneous networks.

- Both transmission and reception are synchronous like using full-duplex technique.

- Applying massive multiple-input multiple-output (massive-MIMO) and millimeter-wave (mm-wave) communications technologies.

- Applying energy-aware communication and energy harvesting to improve energy efficiency.

- Using Cloud Radio Access Network (C-RAN) technology.

- Virtualization of wireless resources.

The 5G networks will consist of massive number of cells. To obtain a multi-tier architecture, these cells must have heterogeneous characteristics and capacities such as macrocells, femtocells and picocells, and using different communication techniques like D2D user equipments UEs.
5G network nodes must have the capability to apply selforganization techniques such as spectrum allocation, autonomous load balancing, power adaptation, interference minimization and others, due to increasing complexity in network management and coordination among the tiers as it is multi-tier network [12]. Furthermore, user equipments UEs must have the ability to connect simultaneously with many base stations or access points by using the same or different radio access technologies (RATs).

The efficiency of overall communication in 5G networks will be improved by integrating many techniques together such as spectrum reuse, three-dimensional or full-dimensional MIMO, network-assisted interference cancellation and suppression, and coordinated multipoint (CoMP) joint transmission and reception [13]. Different devices in the $5 \mathrm{G}$ networks must have the ability to operate in multiple spectrum bands, ranging from radio frequency to $\mathrm{mm}$-wave, while still being compatible with $3 \mathrm{G}$ and $4 \mathrm{G}$.

As mentioned above, one of the most important points to address $5 \mathrm{G}$ networks requirements is to improve energy efficiency, where $5 \mathrm{G}$ provides the service of multimedia applications so it is desirable that the devices integrate energy harvesting technologies and energy-aware communication. Fig. 3 show heterogonous multi-tier $5 \mathrm{G}$ cellular network.

\section{TECHNOLOGIES FOR 5G HETEROGENEOUS NETWORKS}

\subsection{Small Cells}

5G networks will be a multi-tier heterogeneous network which consists of macrocells along with a massive number of low power cells and nodes along with $\mathrm{P} 2 \mathrm{P}$ communication such as D2D and M2M. The deployments of these cells will have much higher density than the existing conventional single-tier networks.

Heterogeneous networks with different classes of base stations, macro-, pico-, and femto- base stations provide a flexible coverage area and improve spectral efficiency. Also 
these different classes of base stations can provide a solution for the growing data traffic.

In $5 \mathrm{G}$ networks the demand for higher data rates will be increased, this can be solved by using the technique of small cells. By reducing the size of the cell, area spectral efficiency will be increased through higher frequency reuse, while the power of transmitting data can be reduced such that the power lost through propagation will be lower. Furthermore, deploying small cells indoors, in office building or home for example, will improve the coverage.

In multi-tier networks the inter-tier and intra-tier interferences are well managed, so the use of such these networks will provide better performance in terms of coverage, capacity, spectral efficiency, and power consumption [14]. These increasing degrees in network cooperation especially between the multiple tiers, from coordination between open network nodes to completely centralized control, will provide increasing levels of network capacity.

Small cells can be different in 5G multi-tier networks, some of them are low powered femtocells which used in residential and enterprise deployments, others are higher powered picocells which used for wider outdoor coverage or filling in macro cell coverage holes .

It is important to remark that mm-wave is recommended to be used in $5 \mathrm{G}$ networks by many researchers and studies as we will show below, so combining mm-wave use with small cells will make the network more reliable. Thanks to the natural evolution to small cells which ensures that frequencies will overcome any attenuation due to rain.

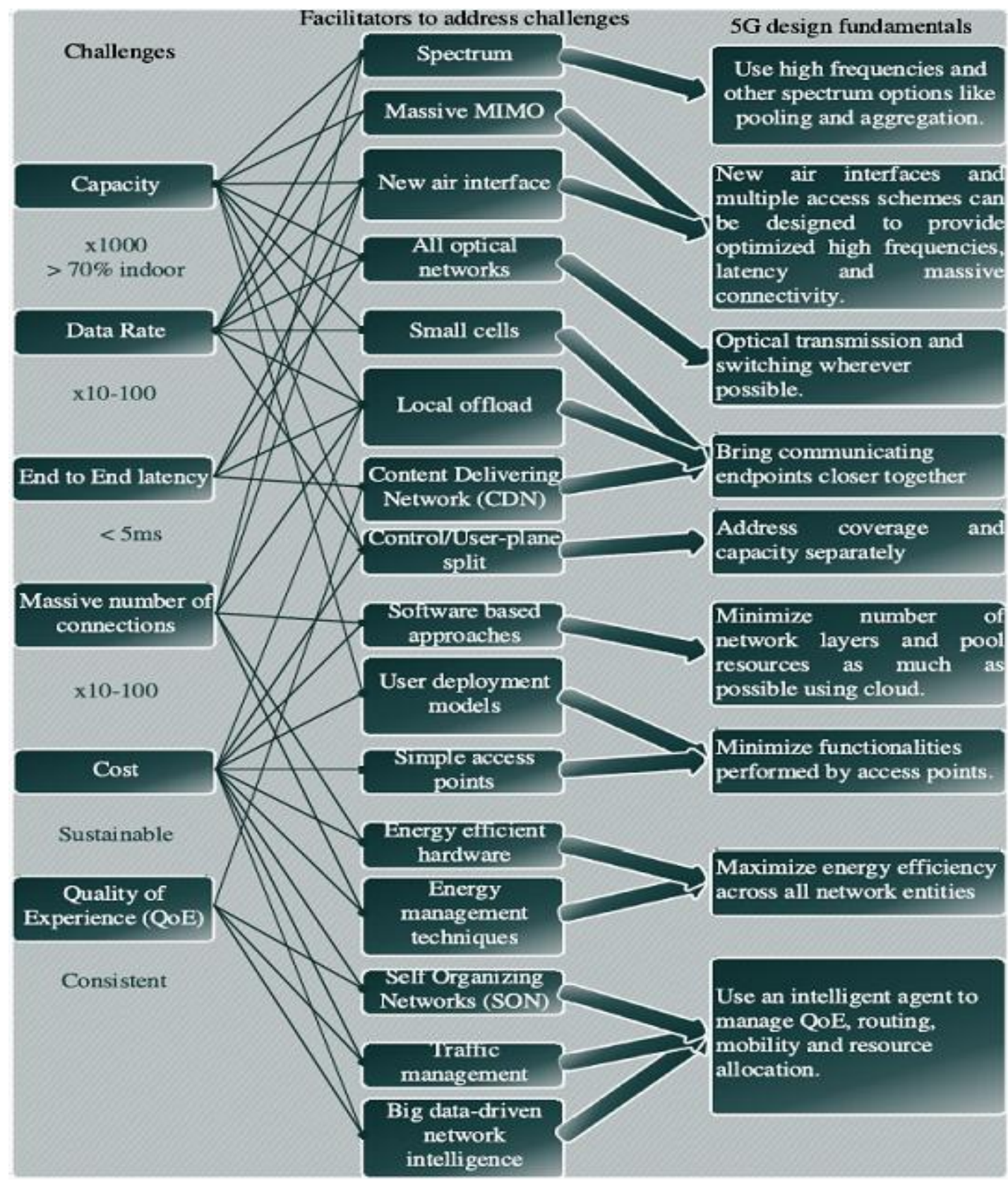

Figure 2 5G Challenges, Facilitators, and Design Fundamentals 


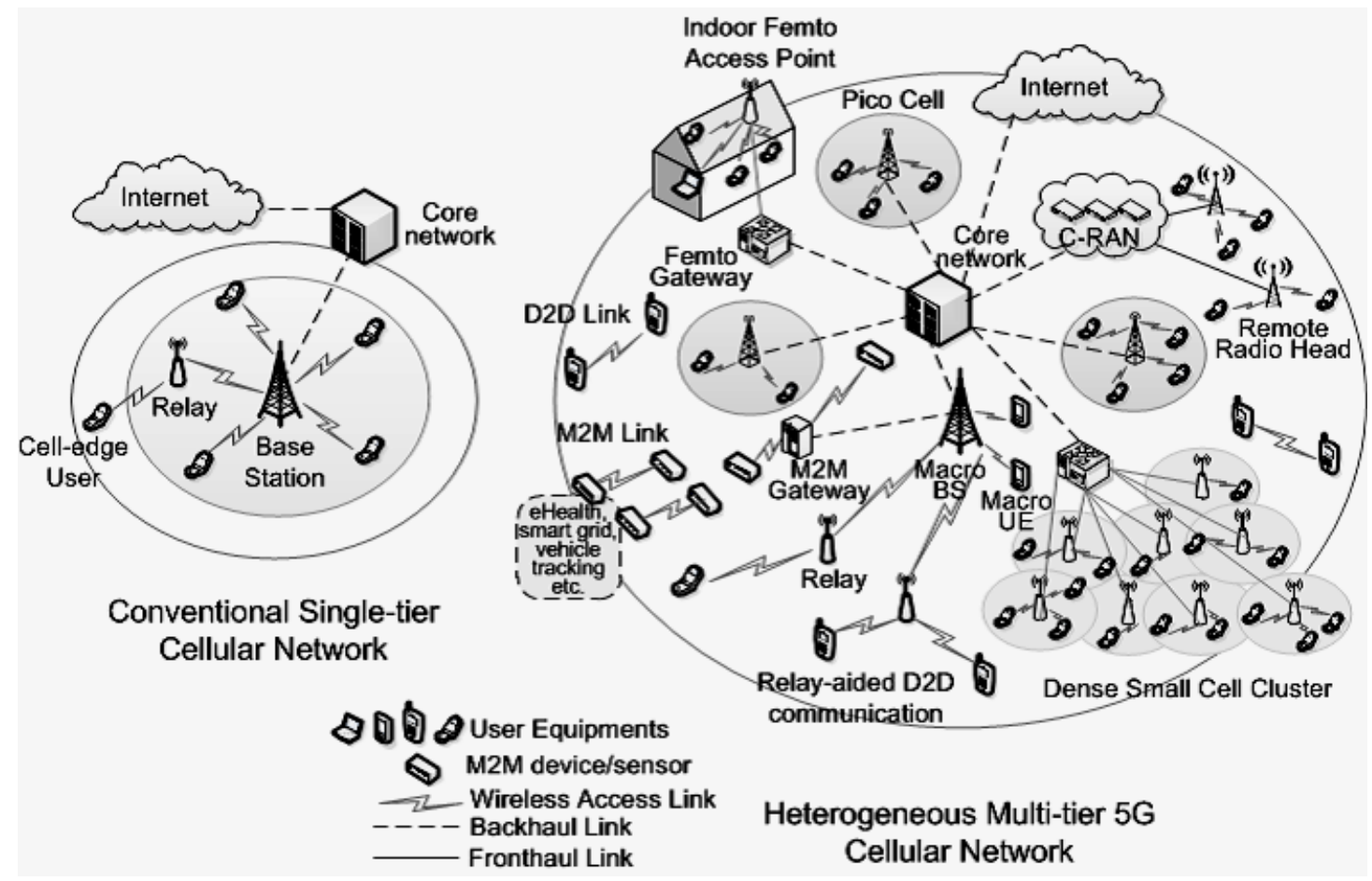

Figure 3 Heterogonous Multi-Tier 5G Cellular Network

\subsection{D2D Communications}

D2D is a new technique to improve network performance in mobile networks by solving the highly dense network problem. This technique enables each terminal (e.g., user equipment UE) to communicate directly with other terminals without the use of base stations, in order to either share their radio access connection, or to exchange information.

In $4 \mathrm{G}$ networks architecture there is no support for direct communication between different devices, all communications will have to be routed through the base station or gateway which is inefficient according to the delay time in receiving data especially when the devices are close by.

This approach is inappropriate for $5 \mathrm{G}$ networks where massive number of cells communicate with each other, especially for multimedia applications. So, it would be more efficient if devices can communicate directly with each other through the concept of D2D to reduce communication delay.

In non-licensed frequency bands, devices can communicate with each other outside of the mobile standard using other technologies such as Bluetooth. However, these connections are liable to interference. While, applying D2D technology reduces interference. Moreover, D2D has additional advantages such as reducing end to end latency, improving cellular coverage, increasing spectral efficiency.

\subsection{Full-Duplex Communication}

Full-Duplex communication (FD) is a communication that allows transceiver to transmit and receive on the same frequency at the same time. In general, wireless cells cannot decode a received data while it is transmitting at the same time on a same frequency band, this because of selfinterference (SI), which means the internal interference between the transmitter and the receiver circuits.
As [15] shows, some techniques can minimize SI in FD systems by combining them together, these techniques are: antenna cancellation, RF interference cancellation, and digital interference cancellation. Interference management is more complex in FD systems due to new interference situations. For a multi-user channel-sharing situation, in addition to intra-cell interference, there are inter-cell downlink-to-uplink interference and inter-cell inter-user uplink-to-downlink interference.

By removing separate frequency band/time slot for both uplink and downlink transmission, FD communication has the prospect to double the spectral efficiency at the physical layer. Comparing with half-duplex communication systems, FD systems can provide higher data rates [16]. Also, FD technology can reduce the latency by synchronously receiving the feedback signals from the receiver during transmission. Furthermore, FD technology can solve wireless networks problems, such as large end-to-end delays, hidden terminals, and loss of throughput due to congestion [11].

\subsection{Millimeter-Waves}

Providing 4G technology with LTE is limited by spectrum availability. LTE uses lots of bandwidth, and carriers only have so much spectrum. The amount of spectrum is limited while the cost of buying new spectrum is high. Small cells as a solution such as Microcells, picocells, and femtocells are limited-range base stations, so they will adopt frequency reuse techniques to provide more efficient use of the spectrum available.

The small cells in 5G network proposed to use mm-wave bands to provide that desirable spectrum needed for expansion. Highly directional antennas with automatic positioning and beamforming make mm-wave frequencies efficient for mobile phones that need great link reliability.

The mm-wave is considered to be the range of wavelengths from 10 millimeters to 1 millimeter, which are longer than 
infrared waves and shorter than radio waves or microwaves and with radio band frequencies of $30 \mathrm{GHz}$ to $300 \mathrm{GHz}$. These high frequencies of mm-waves make them useful in $5 \mathrm{G}$ networks due to their capabilities to transmit large amounts of computer data and mobile applications and communications.

LTE in its future states will have suffer limited spectrum as we mentioned above, while mm-waves have the ability to put hundreds of antennas in a very small space, which allows providing very narrow energy beams.

Rappaport et al, in [17] shows that The combination of costeffective CMOS technology that can work well into mm-wave frequency bands, and steerable antennas at the cells and base station, this combination will support the viability of $\mathrm{mm}$ wave wireless communications. Additionally, mm-wave frequencies allow for larger bandwidth, which means higher data transfer rates.

In mobile radio channels, the data capacity will be greatly increased, and the latency for digital traffic will be decreased by increasing the RF channel bandwidth, as a result, this will support much better internet-based access and applications that require less latency. Mm-wave frequencies, due to the much smaller wavelength, may take advantage of polarization and new spatial processing techniques, such as adaptive beamforming and massive MIMO [18].

In addition, the cost per base station will drop as they become more plentiful and more densely distributed in urban areas, based on that operators continue to reduce cell coverage areas to exploit spatial reuse, and implement new cooperative architectures such as interference mitigation between base stations, relays, and cooperative MIMO. This will lead to make wireless backhaul essential for quick deployment, reduced ongoing operating costs, and flexibility.

Finally, mm-wave spectrum will have much closer spectral allocations than the disjointed spectrum employed these days where the coverage distances of cell sites vary in wide area over three octaves of frequency between $700 \mathrm{MHz}$ and 2.6 $\mathrm{GHz}$. as a result, the propagation characteristics of different mm-wave bands will be more comparable and "homogenous". With the remark that The $28 \mathrm{GHz}$ and 38 $\mathrm{GHz}$ bands are currently available with spectrum allocations of over $1 \mathrm{GHz}$ of bandwidth [19].

One of the most important requirements to develop future mm-wave mobile networks as well as backhaul techniques is the understanding the radio channel.

\subsection{Massive MIMO}

Using a large array of antenna elements which is known as Massive Multiple-Input/Multiple-Output (MIMO), is another technology proposed to be applied in 5G networks to provide diversity and compensate for path loss. Massive MIMO technology is very useful at higher frequencies where can miniaturize antenna elements. Additionally, it allows for high resolution beamforming.

Each antenna element is expected to use extremely low power which will improve energy efficiency. In addition, if channel state information is available, it can exploit large number of degrees of freedom provided by applying MIMO technology in network, by using beamforming [20]. Antenna arrays will be allocated at existing Macro base stations by Massive MIMO base stations, which can concentrate transmitted energy to the mobile users. Massive MIMO deployment can be achieved at different systems even at microwave frequency bands since adequate space is available to do so.
Today's wireless networks operate at microwave frequencies below $6 \mathrm{GHz}$. The frequencies in $5 \mathrm{G}$ network while using $\mathrm{mm}$ wave technology ranging $30 \mathrm{GHz}-300 \mathrm{GHz}$, which can provide huge spectrum.

Using Massive MIMO technology and small cells technique are enhanced by a move to $\mathrm{mm}$-wave spectrum, since the teeny wavelengths allow for hundreds of antenna elements to be placed in an array on a small physical platform at the base station.

A special type of Massive MIMO is 3D MIMO which allows for 3D beamforming. In this technique, it is concerned with using the antenna elements for beamforming. 3D MIMO allows beam control in both vertical and horizontal directions. This additional control allows for further sectorization within a cell. In general, 3D MIMO and full dimensional (FD) MIMO techniques considered techniques that increase the overall network efficiency as they allow mobile networks to support a large number of UEs using multiuser MIMO techniques.

\subsection{Cloud-RAN}

5G networks will be a mixture of different radio access technologies. Compared to the traditional RANs, C-RAN provides many advantages such as scalability and flexibility of deployment a great number of remote radio heads (RRHs), which will be more convenient to 5G networks.

The concept of Cloud Radio Access Network (C-RAN) evolved from a distributed base station architecture where a base station server is responsible for baseband processing. CRAN consists of three main components:

- $\quad$ BBU: refers to BaseBand processing Units which indicates a pool with centralized processors.

- RRHs: refers to Remote Radio Heads which indicates analog radio access units with antennas located at the remote sites.

- Fronthaul network: this network connects RRHs to BBU pool and requires high bandwidth and lowlatency.

The RRHs transmit the RF signals to UEs in the downlink or forward the baseband signals from UEs to the BBU pool for processing in the uplink. RRHs include interface adaptation, filtering, RF amplification, up/down conversion, and A/D and D/A conversion. RRHs can be simple and can be distributed in wide area of the network efficiently, as a result of conducting most of signal processing functions in the BBU pool. The use of inexpensive and densely deployed RRHs in C-RAN will improve network capacity, enhance scalability, and provide the coverage of future $5 \mathrm{G}$ networks.

The BBU pool consists of general purpose processors that operate as virtual base stations to process baseband signals or perform baseband processing. it helps in optimizing the network resource allocation. The backhaul connects the pool with the mobile core network, while the fronthaul spans from the RRHs to the pool for transmitting digital baseband signals.

The fronthaul links can be performed by wired links such as fiber or wireless links, and falls into two categories: ideal without bandwidth constrain and non-ideal. Optical fiber is considered to be an ideal fronthaul for C-RAN, because it can provide large bandwidth and high data rate, while wireless backhauls are faster and cheaper to deploy than fiber. 
C-RAN reduces energy consumption by enabling Massive MIMO or coordinated multipoint (CoMP) concepts. Also, CRAN reduces the cost of baseband processing and reduces the power consumption by performing load balancing and cooperative processing of signals from different BSs. In addition, the number of base stations can be reduced as a result of BBU aggregation in the pool, which results in much lower cost.

\subsection{Energy-Aware Communication and Energy Harvesting}

Energy efficiency of mobiles, cells, and wireless devices in $5 \mathrm{G}$ networks is one of the main challenges in these networks .Harvesting energy from energy sources could be a potential solution to prolong the battery life and also improve the overall energy efficiency of the network, but it may not be feasible for reliable wireless applications, where available energy levels in these resources may vary significantly over time, locations, weather conditions etc.

As another solution, energy can be harvested from ambient radio signals. RF-powered Energy-Harvesting Network (RFEHN) considered being a promising technique for $5 \mathrm{G}$ wireless networks, where the UEs can harvest energy from hybrid access point using RF signals for their information processing and transmission [21]

RF-EHN consists of RF energy sources, these resources can be dedicated RF energy transmitters or ambient RF sources. The Access Points (APs) refer to network nodes such as base stations. AP has an information transmission zone and energy harvesting zone. The energy harvesting zone is smaller than the information transmission zone, since the operating power of the energy-harvesting component is higher than that of the information decoding component [21].

\subsection{Wireless Network Virtualization}

Network virtualization will become one of the main promising technologies in $5 \mathrm{G}$ networks. Wireless Network Virtualization (WNV) together with C-RAN, facilitates resource sharing among many operators.

WNV enables multiple network operators to share resources such as licensed spectrum, energy, Radio Access Network, backhaul, and network infrastructure [22]. The virtualization mechanism isolates the physical resources to be virtual resources, which different service providers are shared.

The main advantages of WNV include: better Quality-ofExperience (QoE) for end users, reduced CAPEX and OPEX, high resource utilization, easier migration to newer technologies by isolating part of the network, improved system performance and [23].

\section{CONCLUSION}

In this paper, the evolution of wireless networks was introduced, 5G heterogonous networks architecture was discussed along with its requirements and challenges, and several emerging technologies for 5G cellular wireless networks were illustrated. In addition to the technologies mentioned above, technologies such as Software-Defined Networking (SDN) and Machine to Machine Communications (M2M) will also impact the development of 5G networks. Therefore, all of the above technologies are proposed to be integrated together to be the potential technologies to meet the requirements of the 5G network and address its challenges. Author aims to do more works in future to provide the facilities and the proper environment to build 5G heterogeneous network with the above technologies, as it is not easy to establish a wireless communication with all previous technologies. Also, more studies and researches will be done to determine demands needed to establish 5G heterogeneous network in Middle East regions.

\section{REFERENCES}

[1] Mohammad Meraj ud in Mir et al, "Evolution of Mobile Wireless Technology from 0G to 5G.", (IJCSIT) International Journal of Computer Science and Information Technologies, Vol. 6 (3) , 2015, pp. 25452551

[2] I. Al-Qasrawi and O. Al-Hazaimeh, "A Pair-wise Key Establishment Scheme for Ad-hoc Networks", International Journal of Computer Networks \& Communications (IJCNC) Vol.5, No.2, pp. 125-136, March 2013.

[3] Ajit Singh, Sharad Nigam, Narendra Gupta, "A Study of Next Generation Wireless Network 6G", International Journal of Innovative Research in Computer and Communication Engineering, Vol. 4, Issue 1, January 2016, ISSN(Online): 2320-9801, pp. 871-874.

[4] T. Halonen, J. Romero, and J. Melero, Eds., "GSM, GPRS and EDGE Performance: Evolution Towards 3G/UMTS". NewYork,NY, USA:Wiley, 2003.

[5] Ms. Anju Gawas, "An Overview on Evolution of Mobile Wireless Communication Networks: 1G-6G", International Journal on Recent and Innovation Trends in Computing and Communication, Volume: 3 Issue: 5, ISSN: 2321-8169, May 2015, pp. 3130-3133.

[6] M. Fallgren et al., Scenarios, "Requirements and KPIs for 5G Mobile and Wireless System", document ICT317669-METIS/D1.1, Apr. 2013.

[7] Industry Proposal for a Public Private Partnership (PPP) in Horizon 2020 (Draft Version 2.1), "Horizon 2020 Advanced 5G Network Infrastructure for the Future Internet PPP". [Online]. Available: http://www.networksetp-eu/_leadmin/user_upload/Home/draft-PPPproposal.pdf

[8] A. Osseiran et al., "Scenarios for 5G mobile and wireless communications: The vision of the METIS project", IEEE Commun. Magazine., vol. 52, no. 5, pp. 26 - 35, May 2014.

[9] P. Demestichas, A. Georgakopoulos, D. Karvounas, K. Tsagkaris, V. Stavroulaki, J. Lu, C. Xiong, and J. Yao, " $5 \mathrm{G}$ on the horizon: Key challenges for the radio-access network," IEEE Vehicular Technology Magazine, vol. 8, no. 3, pp. 47-53, Sept. 2013.

[10] A. Zakrzewska, S. Ruepp, and M. Berger, "Towards converged 5G mobile networks - Challenges and current trends," in Proc. ITU Kaleidoscope Academic Conference, pp. 39-45, Jun. 2014.

[11] S. Talwar, D. Choudhury, K. Dimou, E. Aryafar, B. Bangerter, and K. Stewart, "Enabling technologies and architectures for 5G wireless," in Proc. IEEE MTT-S International Microwave Symposium (IMS), pp. 1-4, Jun. 2014

[12] A. Zakrzewska, S. Ruepp, and M. Berger, "Towards converged 5G mobile networks - Challenges and current trends," in Proc. ITU Kaleidoscope Academic Conference, pp. 39-45, Jun. 2014. 
[13] Q. Li, H. Niu, A. Papathanassiou, and G. Wu, "5G network capacity: Key elements and technologies," IEEE Vehicular Technology Magazine, vol. 9, no. 1, pp.71-78, Mar. 2014.

[14] E. Hossain, M. Rasti, H. Tabassum, and A. Abdelnasser, "Evolution towards 5G multi-tier cellular wireless networks: An interference management perspective," IEEE Wireless Communications, vol. 21, no. 3, pp. 118127, Jun. 2014

[15] J. Choi, M. Jain, K. Srinivasan, P. Levis, and S. Katti. "Achieving single channel, full duplex wireless communication," in Proc. International Conference on Mobile Computing and Networking, pp. 1-12, 2010.

[16] S. Hong, J. Brand, J. Choi; M. Jain, J. Mehlman, S. Katti, and P. Levis, "Applications of self-interference cancellation in 5G and beyond," IEEE Communications Magazine, ISSN: 0163-6804, vol. 52, no. 2, pp. 114-121, Feb. 2014.

[17] T. S. Rappaport, E. Ben-Dor, J. N. Murdock, and Y. Qiao, "38 GHz and $60 \mathrm{GHz}$ Angle-dependent Propagation for Cellular and peer-to-peer wireless communications," in Proc. IEEE Int. Conf. Commun., Jun. 2012, pp. 4568_4573.

[18] F. Rusek, D. Persson, B. Lau, E. Larsson, T. Marzetta, O. Edfors, and F. Tufvesson, "Scaling up MIMO:
Opportunities and challenges with very large arrays," IEEE Signal Process. Mag., vol. 30, no. 1, pp. 40-60, Jan. 2013.

[19] S. Y. Seidel and H. W. Arnold, "Propagation measurements at $28 \mathrm{GHz}$ to investigate the performance of local multipoint distribution service (LMDS)," in Proc. Global Telecommun. Conf., vol. 1. Nov. 1995, pp. 754757.

[20] E.G. Larsson, F. Tufvesson, O. Edfors, T.L. Marzetta, "Massive MIMO for Next Generation Wireless Systems," IEEE Communications Magazine, ISSN: 0163-6804, vol.52 issue: 2, pp. 186-195. Feb. 2014

[21] L. Xiao, P. Wang, D. Niyato, D. Kim, and Z. Han, "Wireless networks with RF energy harvesting: A contemporary survey," IEEE Communications Surveys \& Tutorials, Vol. 17, Issue: 2, pp. 757-789, 2015

[22] E. Hossain and M. Hasan, "5G cellular: key enabling technologies and research challenges", IEEE Instrumentation \& Measurement Magazine, vol. 18, issue. 3, pp. 11 - 21, June 2015

[23] C. Liang and F.-R. Yu, "Wireless network virtualization: A survey, some research issues and challenges," IEEE Communications Surveys \& Tutorials, Vol. 17, Issue: 1, pp. 358-380, 2015 